\title{
Paper No. ICNMM2006-96135
}

\section{EXPERIMENTAL INVESTIGATION OF HEAT TRANSFER AND PRESSURE DROP IN POROUS METAL FOAMS}

\author{
Oliver Reutter/German Aerospace Center, Linder \\ Höhe, 51143 Köln, Germanyloliver.reutter@dlr.de
}

\author{
Elena Smirnova/German Aerospace Center, Linder \\ Höhe, 51143 Köln, Germanylelena.smirnova@dlr.de
}

\author{
Jörg Sauerhering/German \\ Aerospace Center, Linder Höhe, \\ 51143 Köln, Germanyl \\ joerg.sauerhering@dlr.de
}

\author{
Stefanie Angel/Department of \\ Ferrous Metallurgy, Aachen \\ University, 52066 Aachen, \\ Germanylangel@iehk.de
}

\author{
Thomas Fend/German Aerospace \\ Center, Linder Höhe, 51143 Köln, \\ Germany/thomas.fend@dIr.de
}

\author{
Robert Pitz-Paal German Aerospace Center, Linder \\ Höhe, 51143 Köln, Germanyl \\ robert.pitz-paal@dlr.de
}

\begin{abstract}

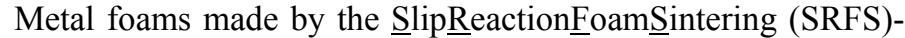
process are investigated. In these foams the pores are produced by a reaction between iron and a weak acid. The generated hydrogen forms pores in a metal powder slurry. These pores remain in the foam after sintering. Also secondary pores are found in these foams because of the sintering of the metal powder slurry. The metal powder base of the foams is Inconel 625 and Hastelloy B. Foam samples with a variety of different porosities of the two metals in the range of about $62 \%$ to $87 \%$ are used as well as samples made out of sintered metal powder which were not foamed with porosities of around $50 \%$.

The motivation for this study is to use these foams as combustion chamber walls in gas fired power plants. By using porous walls effusion cooling can be applied to keep the wall temperatures low.

Air is used as a fluid to study the flow characteristics of these samples. Their pressure drop with air at room temperature is measured in the range of velocities of up to around $1 \mathrm{~m} / \mathrm{s}$. The parameters characterizing the foams are obtained using the Darcy-Forchheimer equations resulting in the permeability and the inertial coefficients. The dependency on the porosity is discussed.

The volumetric heat transfer is measured for the foams by a transient method based on an air flow with a sinusoidal temperature wave, which is attenuated by the sample. The obtained volumetric heat transfer coefficients are discussed and transferred to $\mathrm{Nu}-\mathrm{Re}$ correlations. Correlations between the
\end{abstract}

heat transfer coefficients and the pressure drop coefficients are made.

\section{INTRODUCTION}

The examined samples are made by the SlipReactionFoamSintering (SRFS)-process. This is a promising way to produce open porous metal foams. The advantage of this way of making metal foams is that it is a foaming process which takes place at room temperature and therefore needs only lean machinery. The metal foams can be produced in a great variety of porosity, pore sizes and pore form distributions. Many different metals can be used as the basis of the SRFS-process, which results in many different types of foam.

Open porous metal foams can be applied for many different applications in heat transfer, filter technology and in structural design. The application on which this study in the collaborative research center 'Thermally Highly Loaded, Porous and Cooled Multi-Layer Systems for Combined Cycle Power Plants' aims is the use in the porous wall of a combustion chamber $\left[{ }^{1}\right]$. In order to raise temperatures in the combustion chamber and thereby the efficiency of the combined cycle, the walls have to be cooled. One route of cooling is effusion cooling and the application of the SRFSfoams is investigated for this purpose. In order to design the effusion cooling and to calculate the thermal behavior numerically $\left[{ }^{2}\right]$ it is necessary to study the behavior of the foams under gas flow. 


\section{NOMENCLATURE}

Latin:

A Crosssection of the sample $\left[\mathrm{m}^{2}\right]$

$A_{V} \quad$ Specific inner surface $\left[\mathrm{m}^{2} / \mathrm{m}^{3}\right]$

c Heat capacity of the fluid $[\mathrm{J} / \mathrm{kg} \mathrm{K}]$

$\mathrm{d}_{\mathrm{h}} \quad$ Hydraulic diameter [m]

$\mathrm{dp} \quad$ Pressure drop factor [-]

$\mathrm{K}_{1} \quad$ permeability [m²]

$\mathrm{K}_{2} \quad$ inertial term [m]

$\mathrm{L} \quad$ Length of the sample [m]

$\dot{M} \quad$ massflow $[\mathrm{kg} / \mathrm{s}]$

$\mathrm{Nu} \quad$ Nußelt-number [-]

$\mathrm{p} \quad$ Pressure $[\mathrm{Pa}]$

Pr Prandtl-number [-]

$\mathrm{Re} \quad$ Reynolds-number [-]

$\mathrm{T} \quad$ Temperature [K]

$\mathrm{t} \quad$ Time [s]

v Velocity $[\mathrm{m} / \mathrm{s}]$

$\mathrm{x} \quad$ Coordinate $[\mathrm{m}]$

Greek:

$\alpha \quad$ Heat transfer coefficient $\left[\mathrm{W} / \mathrm{m}^{2} \mathrm{~K}\right]$

$\eta_{\text {Dyn }} \quad$ Dynamic viscosity of the fluid [Pa s]

$\lambda \quad$ Heat conductivity of the fluid[W/m K]

$\rho \quad$ Density of the fluid $\left[\mathrm{kg} / \mathrm{m}^{3}\right]$

\section{SAMPLE SPECIFICATION}

The samples are made by the SRFS-process $\left[{ }^{3}\right]\left[{ }^{4}\right]$. In this process a metal powder is mixed with a dispersant, a solvent and phosphoric acid. The reaction between the metal particles and the phosphoric acid forms a metal phosphate and releases hydrogen. This hydrogen foams the slip made of the metal powder and the solvent. In case the metal does not react with the phosphoric acid an iron powder is mixed with the metal powder as an expanding agent. The metal phosphate freezes the hydrogen bubbles in the slip. The slip is cast into the required form. The samples are dried and then sintered under reducing conditions in order to reduce the metal phosphate and avoid oxidation.

The investigated samples are made of Hastelloy B powder and Inconel 625 powder. The powder sizes range between 50 and $150 \mu \mathrm{m}$.

Samples made only of the sintered slip without foaming show a porosity of about $50 \%$. These samples are taken as representing the material of the pore walls in the foams. The foams have a total porosity between 62 and $87 \%$. The pores can be characterized as those which arise from the hydrogen foaming, called the primary pores, and those which are the pores which come from sintering the slip, called the secondary pores. The secondary pores reach up $0.3 \mathrm{~mm}$ diameter and the primary pores reach up to $3.5 \mathrm{~mm}$.

The samples were produced at the Department of Ferrous Metallurgy at Aachen University. The samples are cylinders with diameters between 78 and $81 \mathrm{~mm}$ and the length was between 9 and $30 \mathrm{~mm}$. The picture of a sample can be seen in figure 1 .
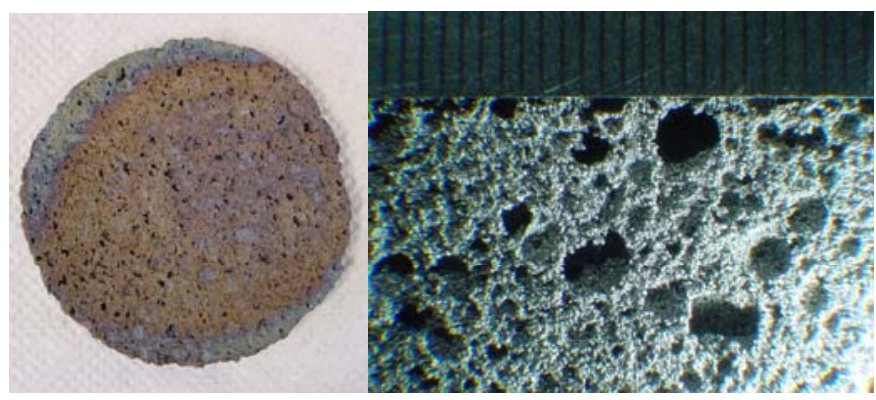

Figure 1: Picture of a sample of a Hastelloy B foam and a detailed view of the pore structure together with a millimeter scale.

\section{EXPERIMENTAL SET-UP}

The density of the foams and thereby the porosity is determined by measuring the dimensions of the sample and by weighing.

The pressure drop is measured by an apparatus which first measures the mass flow through the samples and then the pressure difference before and after the sample when subject to flow. The pressure tap on each side of the sample consists of eight holes distributed evenly across the perimeter of the tube. The mass flow is determined by measuring the pressure drop of a well defined orifice. The sample is tightly fitted in a stainless steel tube by surrounding it with several layers of foamed rubber. A blower drives the air through the apparatus. Figure 2 shows a sketch of the experimental set-up. This set-up resembles the situation in the intended application as an effusion cooling wall.

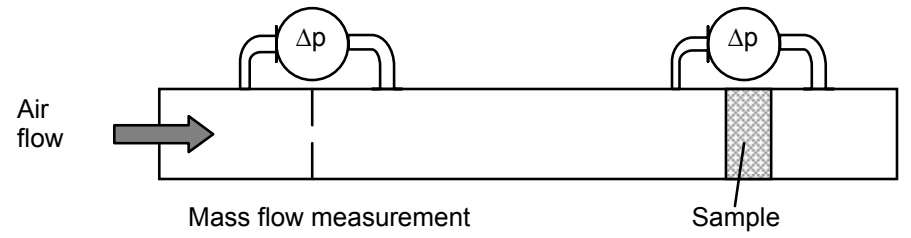

Figure 2: Sketch of the experimental set-up used to measure the pressure drop of the metal foams.

The volumetric heat transfer is measured by an experimental set-up shown in Figure 3. It was constructed for the characterization of cellular materials for the application as receivers for concentrated solar radiation $\left[{ }^{5}\right]$. The experiment is based on a modification of the method by Younis and Viskanta $\left[{ }^{6}\right]\left[{ }^{5}\right]$. An air flow with a sinusoidal alternating temperature is driven through the porous sample. The air temperature can be seen as a temperature wave $T(t, x) . x$ denotes the direction of the air flow and the time. This temperature wave is generated by an electrically driven heat element. The air flow is maintained by a blower. The mass flow is determined in the same way as for the pressure drop measurements by measuring the pressure drop over a defined orifice. It is also possible to control the amplitude and the frequency $\omega$ of the temperature wave of the air flow. The tube through which the air flows is insulated. The air temperature is measured at the entrance $(T(t, 0))$ and at the outlet $(T(t, L))$ of the sample. The sample 
induces a phase shift and an attenuation of the temperature wave. From these, the product of the specific surface $A_{V}$ and the heat transfer coefficient $\alpha$ can be calculated. The product describes how much heat is transferred from the solid to the air in a given volume of foam. In applications like effusion cooling or solar energy conversion in absorbers this product is the relevant parameter, because the heat transfer takes place in the volume.
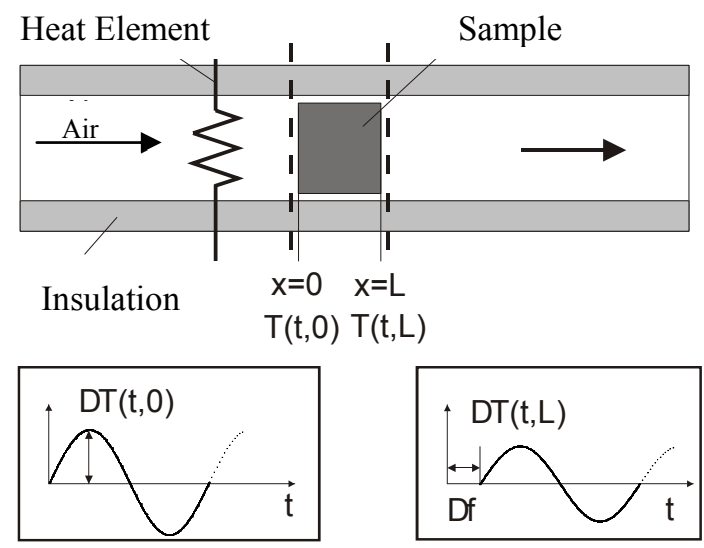

Figure 3: Experimental set-up of the apparatus for measuring volumetric convective heat transfer. The Temperature wave at $\mathrm{x}=0$ and $\mathrm{x}=\mathrm{L}$ is shown.

\section{RESULTS AND DISCUSSION}

Referring to previous publications like those of Innocentini $\left[{ }^{7}\right]$ and Boomsma $\left[{ }^{8}\right]\left[{ }^{9}\right]$ it is adequate to describe the flow through a porous medium in terms of Darcy's equation with the Forchheimer extension and not only by the Darcy's equation. This holds especially if the flow through the porous medium is not very low. Furthermore the experimental data measured in this study fits very well to the Forchheimer Extension, so it was applied for analysis throughout this study:

$$
\frac{\Delta p}{L}=\frac{\eta_{\text {Dyn }}}{K_{1}} v+\frac{\rho}{K_{2}} v^{2}
$$

As the velocity for extracting the flow characteristics the superficial velocity $\mathrm{v}$ is used, that is the velocity which one would have, if the sample had a porosity of $100 \%$. This means that the velocity is based on the mass flow, the density of the air and the cross-section of the sample.

$$
v=\frac{\dot{M}}{\rho A}
$$

A typical measurement of the pressure drop against the velocity in the foam is shown in figure 4 . The quadratic dependency of the pressure drop on the flow velocity can be clearly seen. A quadratic fit on these curves was used. The permeability and the inertial coefficient were calculated from the obtained mathematical coefficients using the temperature at the sample, which was basically room temperature.

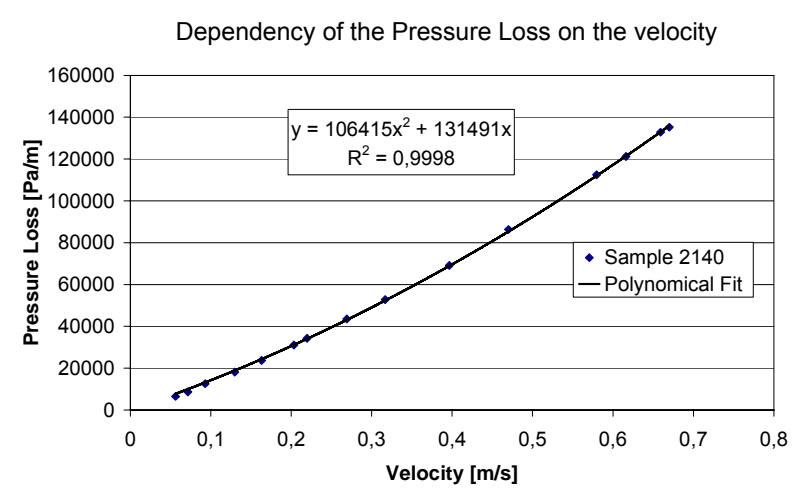

Figure 4: A typical result of a pressure loss measurement. The data is well fitted by a quadratic polynomial fit.

In figure 5 the permeabilities and the inertial coefficients of the Inconel 625 foam samples are plotted versus the density of the sample. This corresponds to a plot versus the porosity. The density of Inconel 625 is $8,440 \mathrm{~g} / \mathrm{cm}^{3}$. The densest samples were not foamed, but samples which were made by only sintering the Inconel powder without adding a foaming agent. The pressure drop of these samples was so high, that the massflow and the velocities through the sample were very low and could not be well measured by the used orifice. Therefore the resulting high error made it impossible to determine the permeability coefficient. A trend can be seen, that samples with higher densities and lower porosity show lower permeability and lower inertial coefficients.

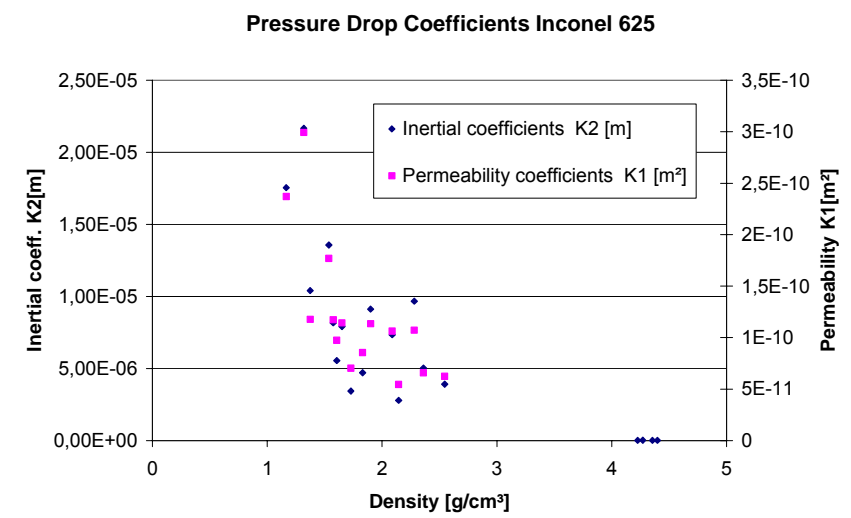

Figure 5: Inertial coefficients and permeability of Inconel 625 samples versus their density. The group of samples with densities over $4 \mathrm{~g} / \mathrm{cm}^{3}$ represents the sintered slip samples without foaming. 
Pressure Drop Coefficients Hastelloy B

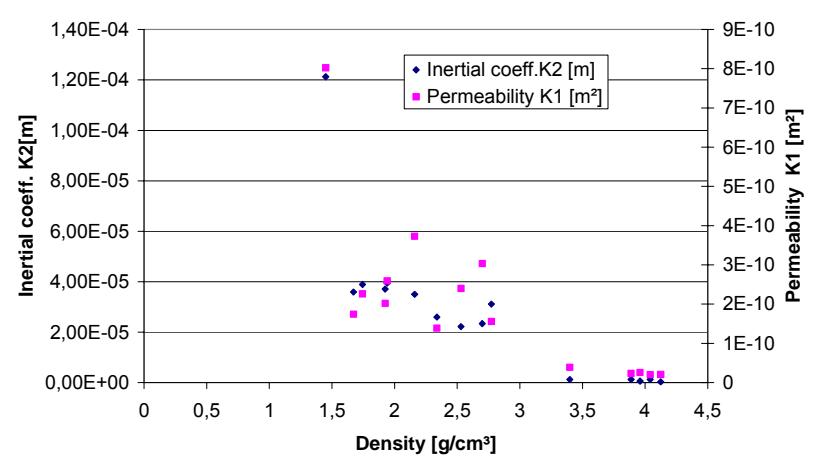

Figure 6: Inertial coefficients and permeability of Hastelloy B samples versus their density. The group of samples with densities from around 3.5 to over $4 \mathrm{~g} / \mathrm{cm}^{3}$ represents the sintered slip samples without foaming.

In figure 6 the permeability and the inertial coefficients of the Hastelloy B samples are shown. The density of the Hastelloy B is $9,220 \mathrm{~g} / \mathrm{cm}^{3}$. Here as for the Inconel 625 samples the densest samples were not foamed but only sintered. The same trend to higher permeability and inertial coefficients with higher porosity can be observed.

Because this material is relatively new and not yet well characterized the density is taken as the governing parameter, because it is well defined. The scatter in the data, the possibility to find a low density sample with a permeability comparable to samples with a significant higher density, clearly shows that also the pore structure must have an influence on the pressure drop. Research to characterize the foams also with respect to pore diameter and specific surface area are ongoing and might enhance the understanding of the dependencies of the pressure drop results.

Volumetric heat transfer measurements on Hastelloy B foam samples are shown in figure 7. The measurement gives the total volumetric heat transfer, which is the product of the heat transfer coefficient $\alpha$ and the specific inner surface $A_{V}$ of the foam. As in the pressure loss measurements the air velocity is used which assumes no sample to be present. The data shows an in principal linear dependency of the volumetric heat transfer on the velocity. The higher the porosity, the lower the volumetric heat transfer.
Hastelloy B Foams

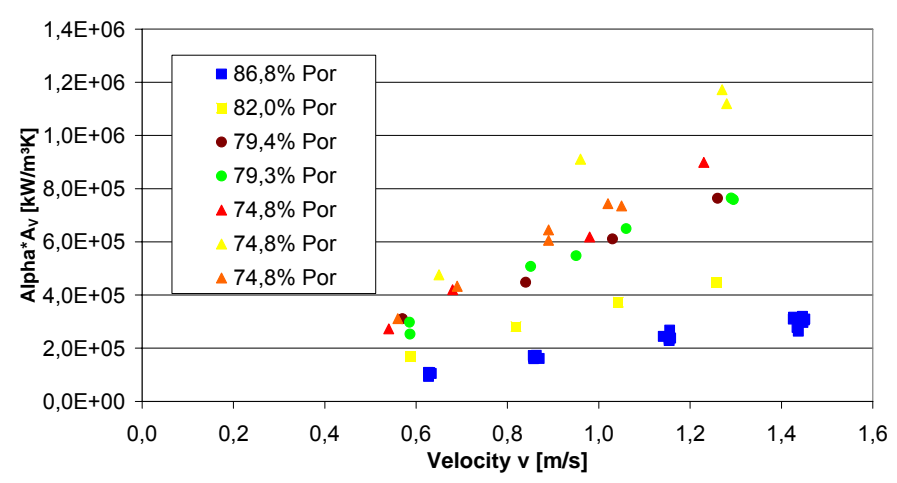

Figure 7: Volumetric heat transfer measurements of Hastelloy $B$ foams versus the velocity of the air flowing through the foam.

Making use of the generalization of these measurements by using the Nußelt-number

$$
N u=\frac{\alpha d_{h}}{\lambda},
$$

the Reynoldsnumber

$$
\operatorname{Re}=\frac{v d_{h} \rho}{\eta}
$$

and the Prandtl-number

$$
\operatorname{Pr}=\frac{\eta c}{\lambda}
$$

these measurement data can be transformed using the properties of air $\left[{ }^{10}\right]\left[{ }^{11}\right]$. For the description of the foam a hydraulic diameter of $0.5 \mathrm{~mm}$ was used. This certainly can be refined in the progress of further research and with a better characterization of the foam material. The order of magnitude for the effective diameter for these pore structures is indicated by analyzing micrographs of the foam and by capillary measurements. The resulting graph can be seen in Figure 8 .

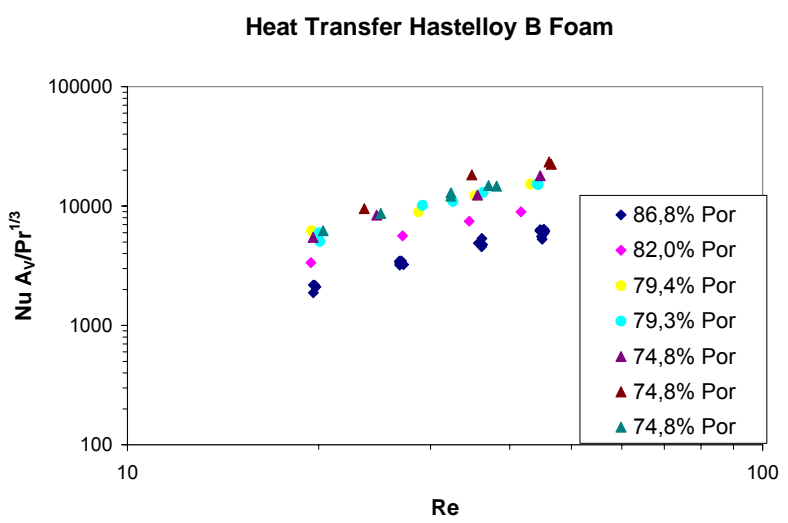

Figure 8: Volumetric heat transfer versus the Reynolds number

Following an approach by Schlünder $\left[{ }^{12}\right]$ and Martin $\left[{ }^{13}\right]$, based on the theory of Leveque, the heat transfer is proportional to the 
pressure drop through a structure. The pressure drop is represented by $\mathrm{dp} \cdot \mathrm{Re}^{2}$ with a pressure drop factor $\mathrm{dp}$ :

$$
d p=0.92 * \frac{d_{h}}{K_{2}} .
$$

The plot of this can be seen in figure 9. A correlation between pressure drop and heat transfer can be seen. The correlation can be expressed as

$$
\frac{N u A_{V}}{\operatorname{Pr}^{\frac{1}{3}}}=10 \cdot\left(d p \operatorname{Re}^{2}\right)^{0.729} \frac{1}{m} \text {. }
$$

This is useful for applying the metal foams in a defined way. For the application as a porous wall for effusion cooling a high heat transfer is desirable in order to transfer a lot of heat from the wall to the cooling air and thereby get a good cooling efficiency. On the other hand a high pressure drop is not desirable because of the energy needed to force the air through the foam.

\section{Hastelloy B}

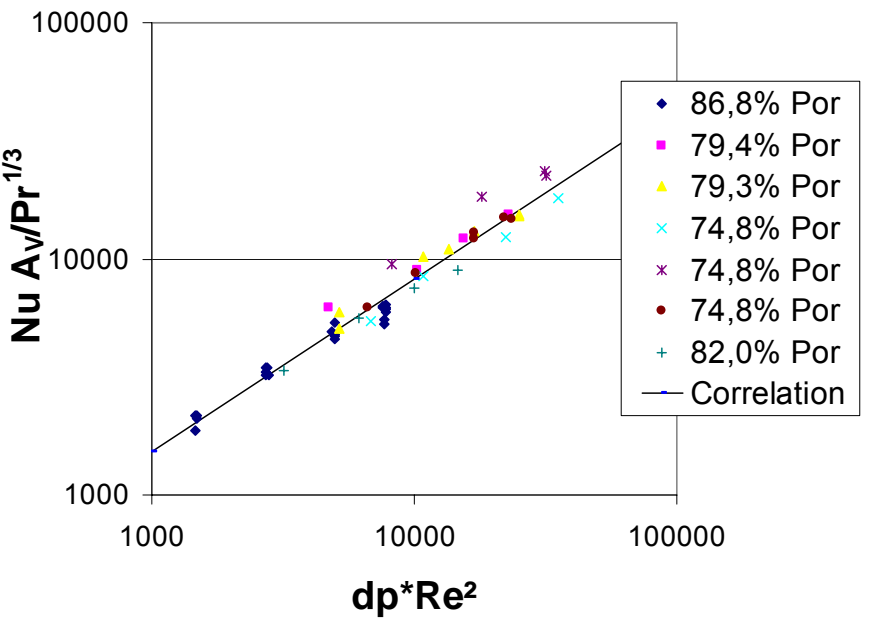

Figure 9 Correlation between heat transfer and pressure drop for Hastelloy B foams.

In a similar way, measurements of Inconel 625 foams are treated. The results are shown in figure 10. The correlation shown in the graph is given by

$$
\frac{N u A_{V}}{\operatorname{Pr}^{\frac{1}{3}}}=10 \cdot\left(d p \operatorname{Re}^{2}\right)^{0.596} \frac{1}{m} \cdot
$$

The exponent of the dependency of the heat transfer on the pressure drop is a different one than for the Hastelloy foams, but the principal behavior is the same.

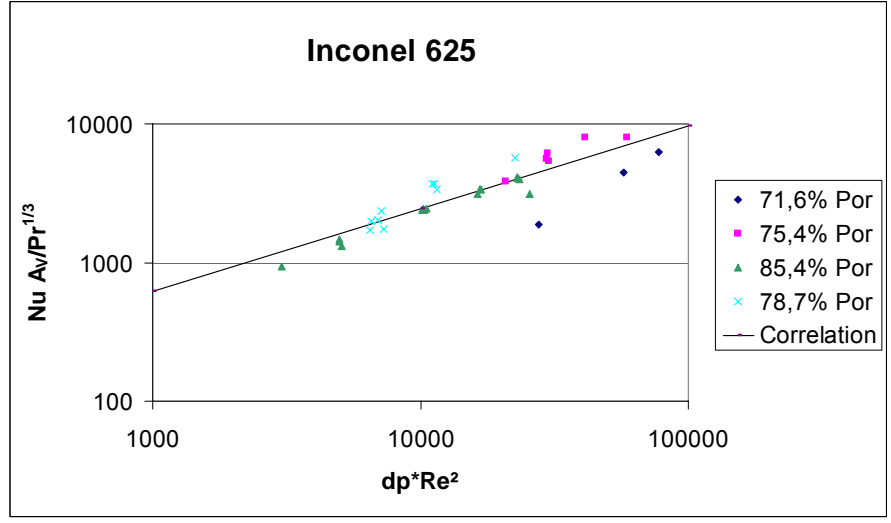

Figure 10 Correlation between heat transfer and pressure drop for Inconel 625 foams.

\section{CONCLUSIONS}

For metal foams made by the SRFS-process based on Hastelloy B and Inconel 625 powder of different porosities measurements of the pressure drop and volumetric heat transfer have been conducted.

As one would expect the foams with a higher density show a higher pressure drop. The foams with higher pressure drop also show higher values of volumetric heat transfer. A correlation between the pressure drop and the heat transfer can be found. Further research will show better correlations and a model for these kinds of foams.

As this material is relatively new and not yet well characterized, there is still a lot of research and model refinement to be done. These foams are not directly comparable to foams produced by other foaming processes, which give more reticulate structures.

These measurements together with the measurements of the thermal conductivity in the same project will give the basis for thermophysical modeling of SRFS metal foams. This knowledge is useful in the design of combustion chamber walls cooled by effusion cooling and other applications of SRFSfoams.

\section{ACKNOWLEDGMENTS}

The support of the Deutsche Forschungsgemeinschaft (DFG) for the collaborative research center 'Thermally Highly Loaded, Porous and Cooled Multi-Layer Systems for Combined Cycle Power Plants' (SFB 561) is thankfully acknowledged.

\section{REFERENCES}

$\left.{ }^{1}\right]$ D. Bohn, New Materials and Cooling Systems for High Temperature, Highly Loaded Components in Advanced Combined Cycle Power Plants, 7th Liege Conference on "Materials for Advanced Power Engineering", Sept. 30 - Oct. 02, 2002, Liege, Belgium

$\left.{ }^{2}\right]$ D. Bohn, N. Moritz, Numerical Investigation on Flow Field and Heat Transfer Phenomena in Multi-Hole Cooling Configurations RTO-Symposium, May 2001, Loen. 
[3] S. Angel, W. Bleck, P.-F. Scholz and Th. Fend, Influence of powder morphology and chemical composition on metalic foams produced by SlipReactionFoamSintering (SRFS)process, Steel Research Int. 75 (2004) 483-488

$\left[{ }^{4}\right]$ S. Angel, W. Bleck, P.-F. Scholz: Adjusting the pore structure of open porous metallic foams produced by the SlipReactionFoamSintering (SRFS)- Process. 4th International Conference MetFoam, September 21-23, 2005, Kyoto, Japan. Proceedings: Japan Institute of Metals

[5] Pitz-Paal R, Hoffschmidt B, Böhmer M, Becker M. Experimental and numerical evaluation of the performance and flow stability of different types of open volumetric absorbers under non-homogeneous Irradiation. Solar Energy 1997, Volume 60, Issues. p 135-150

$\left[{ }^{6}\right]$ Viskanta, R., Younis, L.B. Experimental determination of the volumetric heat transfer coefficient between stream of air and ceramic foam, Int. J. Heat and Mass, Vol 36, No 6, 1425-1434, 1993

$\left.{ }^{7}\right]$ M.D. de Mello Innocentini, P. Sepulveda and F. dos Santos Ortega, Permeability, p 313-341, in M. Scheffler and P. Colombo, Cellular Ceramics, Wiley-VCH, Weinheim, 2005

$\left[{ }^{8}\right]$ K. Boomsma, D. Poulikakos, Y. Ventikos, Simulation of Flow through Open Cell Metal Foams Using an Idealized Periodic Cell Structure, International journal of Heat and Fluid Flow, 24, p. 825-834, 2003

[9 ] K. Boomsma, D. Poulikakos, The Effects of Compression and Pore Size Variations on the Liquid Flow Characterisitcs in Metal Foams, Journal of Fluids Engineering, Vol. 124, p.263272,2002

$\left[{ }^{10}\right]$ VDI-Wärmeatlas 5.Auflage 1988

$\left.{ }^{11}\right]$ E.-U. Schlünder, E. Tsotsas, Wärmeübertragung in Festbetten, durchmischten Schüttgütern und Wirbelschichten, Georg Thieme Verlag Stuttgart, 1988

$\left[{ }^{12}\right]$ E-U. Schlünder,Chemie-Ing.-Techn. 42, p. 905-910, 1970

$\left.{ }^{13}\right]$ H. Martin, V. Gnielinski, Proc. 3rd European Thermal Sciences Conf., p. 1155-1160, 2000 